\title{
COMPARISON BETWEEN METHODS OF ASSESSING LUMBOSACRAL CURVE OBTAINED BY RADIOGRAPHIC IMAGE
}

\author{
Dalane Aparecida $V_{a C a r l}{ }^{1}$, Eduardo Borba Neves ${ }^{1}$, Leandra Ulbricht ${ }^{1}$
}

\section{ABSTRACT}

Objective: To investigate the correlation between different radiographic methods in the evaluation of the lumbosacral concavity. Methods: The sample consisted of 52 individuals with ages ranging from 18 to 28 years old. The procedures related to radiographic image collection were carried out in collaboration with a diagnostic imaging center of a hospital in Curitiba, PR, Brazil. The angles of the lumbosacral concavity were evaluated by the following methods: Centroid, Cobb1 $1_{\text {L1-S1 }}$, Cobb2 $2_{\text {L1-L5 }}$, Cobb3 $_{L 2-S 1}$ Cobb4 $_{T 12-S 1}$ Posterior Tangent and Trall. Results: High correlation coefficients ( $r$ ranging from 0.77 to 0.89 ) were found among variations of the Cobb method. Additionally, we propose a categorical classification of angle values obtained by each method. We also analyzed the influence of the level of the inflection point between the lumbar lordosis and thoracic kyphosis in determining the evaluation method to be used. The inflection point had a higher incidence in the region between the twelfth thoracic vertebra and the first lumbar vertebra (63.5\%). Conclusion: The correlation and agreement between methods vary considerably. Moreover, the thoracolumbar inflection point should be considered when choosing the method of assessing patients. Level of Evidence I, Diagnostic Study.

Keywords: Spine. Lumbosacral region. Radiography. Evaluation.

Citation: Vacari DA, Neves EB, Ulbricht L. Comparison between methods of assessing lumbosacral curve obtained by radiographic image. Acta Ortop Bras. [online] 2015;23(2):67-71. Available from URL: http://www.scielo.br/aob.

\section{INTRODUCTION}

Formed by a complex anatomical structure, the lumbosacral spine is a very researched segment in the scientific midst. ${ }^{1,2}$ The demands arising from the increasing number of cases related to back pain and affections in the lumbosacral structure are usually from poor posture, and in most cases arising from the lack of adaptation of work positions in labor activities, inactivity or congenital and chronic illnesses ${ }^{3}$. In order to prevent the disorders of the lumbosacral spine, the pattern of postural alignment considered optimal should have characteristics of bone muscle, ligament, tendon and joint uniformity, and besides, it should present the proper functioning of the nervous and labyrinthic system. ${ }^{2}$ Due to the constant demands of scientific knowledge related to the spine, many techniques have been developed to analyze and diagnose cases of posture abnormalities. The currently most efficient are X-ray and Magnetic Resonance Imaging. ${ }^{4}$ On the evolution of capture techniques, we observe the progress of the methods aimed at measuring radiographic images. ${ }^{5}$ In order to be reliable, diagnosis regarding this type of image ought to use methods with high levels of inter-rater reliability. Currently, tests like Cobb, Trall, Cen- troid and Posterior Tangent have such characteristics that demonstrate correlatable results, as showed by the study of Lee et al. ${ }^{6}$

However, statistical investigations between the various existing methods for the measurement of lumbosacral curvature remain restricted. Statistical inference addressed in most studies is related to the investigation of reliability intra and interevaluaters. ${ }^{6}$ Therefore, the motivation of this study was to correlate and verify the agreement between the following methods: Cobb, Centroid, Trall, and Posterior Tangent and to propose a categorical classification of the methods studied.

\section{MATERIALS AND METHODS}

The methodology for the design of this study was observational, quantitative descriptive and transversal. In total, 58 individuals volunteered to collection, male and female, aged 18-28 years old. Regarding inclusive requirements for the selection, it was determined that participants would fit between the ages 18 to 35 years old, present a morphological profile compatible with the result of the waist/hip ratio (WHR) ranked from low to moderate, had undergone an assessment by an orthopedic doctor

All the authors declare that there is no potential conflict of interest referring to this article.

\footnotetext{
1. Universidade Tecnológica Federal do Paraná (UTFPR), Campus Curitiba, Curitiba, PR, Brazil.
}

Work developed at Hospital Geral do Exército Brasileiro de Curitiba, Curitiba, PR, Brazil.

Correspondence: Rua Marquês do Paraná 418, apto. 104, Água Verde, Curitiba, PR, Brazil. borbaneves@hotmail.com 
for indication of radiograph exam and delivered the Free and informed consent form duly signed.

Individuals who had undergone some type of radiographic examination in the last year, participants who were not selected by the evaluation of the orthopedic doctor and women, specifically, who were pregnant or nursing were not included in the data collection. Due to the exclusionary factors, the final sample included 52 individuals.

The procedures of radiographic collection were carried out in partnership with a diagnostic imaging center of a hospital in the capital of Curitiba, PR, Brazil. Radiographies of the lumbosacral spine were performed by a radiology professional, at the profile $(P)$ and anteroposterior (AP) incidence and stored in digital format for further analysis. As investigative criteria of this research, we used only images taken in profile, and other images captured in AP were used for diagnosis of orthopedic surgeon applicant.

This research project was submitted to the Research Ethics Committee of Centro Universitário Campos de Andrade and was approved under protocol $\mathrm{N}^{\circ} 438$.

In the radiographic image capture process the equipment used were: a Buck Mural Philips Optimus emitter, a Philips digital control desk, a Regius radiographic film and Computer Radiography (CR) system and the Konica Minolta manager software, which enabled the editing of images taken by CR. Radiographic exams were performed in the afternoon, always by the same professional. The postures adopted by the participants were standardized according to the obtained incidences.

The 1.46r ImageJ software was used for determining the lumbosacral angle according to the following methods: the four variations Cobb ( $\mathrm{Cobb}_{\mathrm{L1}-\mathrm{S} 1} ; \mathrm{Cobb}_{\mathrm{L1}-\mathrm{L} 5} ; \mathrm{Cobb}_{\mathrm{L2}-\mathrm{S} 1}$ e Cobb $\left.\mathrm{Cob}_{\text {T12-S1 }}\right)$; centroide; Trall; and posterior tangent.

\section{Statistical Analysis}

The sample was characterized by descriptive statistical treatment, with the following indicators: minimum value, maximum value, mean, standard deviation and sample size. The inferential statistical analysis started from the Kolmogorov-Smirnov normality test to verify the normality of the distribution of the study variables, with significance level 95\% $(p<0.05){ }^{7}$

Once the data were tabulated, the Pearson linear correlation coefficients between the studied protocols were calculated. After that, each evaluation protocol of the lumbosacral angle was stratified into five categorical tracks, from the value of the means and standard deviations found. (Table 1)

Then the agreement intensities between the classifications of the protocols studied were evaluated using the Kappa agreement coefficient. ${ }^{8}$

Table 1. Classification of angular values.

\begin{tabular}{c|c}
\hline Interval & Category \\
\hline$<(M-2 S D)$ & Far below \\
\hline$(M-2 S D) a(M-1 S D)$ & Moderately below \\
\hline$(M-1 S D) a(M+1 S D)$ & Average \\
\hline$(M+1 S D) a(M+2 S D)$ & Moderately above \\
\hline$>(M+2 S D)$ & Far above \\
\hline
\end{tabular}

M: Mean; SD: Standard deviation.

\section{RESULTS}

The sample consisted of 52 individuals, of which $65.4 \%(n=34)$ corresponded to male subjects and $34.6 \%(n=18)$ to female. The variables related to body weight and height corresponded to the mean and standard deviation of $68.9 \pm 10.1 \mathrm{~kg}$ and $172.9 \pm 8.9 \mathrm{~cm}$ and the mean age was $20.7 \pm 2.6$ years old.

From the sample group chosen for the study, the Pearson correlation test was applied to all methods employed. Table 2 shows the correlation coefficient, its statistical significance and the correlation of the intensity rating for all methods.

In addition to the linear correlation test the angle values found in each method were classified into five categories: far below; moderately below; average; moderately above and far above. The limits of each categorical group were established from the mean values and standard deviations obtained for each method. (Table 3)

The interpretation proposed by Landis and Koch, ${ }^{8}$ to categorize Kappa statistic values, whose values and agreement intensities are shown in Table 4.

The ratings of the angles from Cobb1 and Cobb2 methods showed strong agreement and the highest Kappa index - 0.634 - between all methods.

Besides the proposed categorization process for methods of measurement of lumbar curvature, we identified the vertebrae that presented a higher frequency on the inflection point.

For measuring such a procedur it required the Straight tool and Image J software for the design of the upper end plate of the last thoracic vertebra and first lumbar vertebra. After determining each of these structures, the results of the slopes of each

Table 2. Pearson correlation coefficients between radiographic methods

\begin{tabular}{|c|c|c|c|c|c|c|c|}
\hline & $\mathrm{Cobb}_{2 \text { L1s_L5i }}$ & $\mathrm{Cobb}_{3 \text { L2s_S1s }}$ & $\mathrm{Cobb}_{4 \text { T12i_S1s }}$ & Centroide & Posterior L1xL5 & Posterior L1XS1 & Trall \\
\hline $\mathrm{Cobb}_{1 \text { L1s_S1s }}$ & $0.809^{\star *}$ & $0.810^{\star *}$ & $0.893^{\star \star}$ & $0.611^{\star \star}$ & $0.467^{* *}$ & $0.548^{* *}$ & $0.693^{\star *}$ \\
\hline $\mathrm{Cobb}_{2 \mathrm{~L} 1 \mathrm{~s} \_L 5 i}$ & & $0.800^{* *}$ & $0.820^{* *}$ & $0.664^{* *}$ & $0.502^{* *}$ & $0.603^{* *}$ & $0.707^{* *}$ \\
\hline \multirow{2}{*}{$\mathrm{Cobb}_{3 \text { L2s_S1s }}$} & & & $0.765^{\star *}$ & $0.572^{* \star}$ & $0.444^{\star \star}$ & $0.627^{\star *}$ & $0.677^{\star \star}$ \\
\hline & & & Strong & Moderate & Moderate & Moderate & Moderate \\
\hline \multirow{2}{*}{$\mathrm{Cobb}_{4 \text { T12i_S1s }}$} & & & & $0.585^{\star \star}$ & $0.451^{\star \star}$ & $0.618^{\star \star}$ & $0.651^{\star \star}$ \\
\hline & & & & Moderate & Moderate & Moderate & Moderate \\
\hline \multirow{2}{*}{ Posterior ${ }_{L 1 \times L 5}$} & & & & & & $0.631^{* *}$ & $0.474^{\star \star}$ \\
\hline & & & & & & Moderate & Moderate \\
\hline \multirow{2}{*}{ Posterior $_{L 1 \times S 1}$} & & & & & & & $0.360^{\star *}$ \\
\hline & & & & & & & Weak \\
\hline
\end{tabular}


Table 3. Categorical classification of angular values according to lumbar curvature identification method.

\begin{tabular}{|c|c|c|c|c|c|c|c|c|}
\hline Classification & $\mathrm{Cobb}_{3 \mathrm{~L} 2 \mathrm{~s}-\mathrm{S} 1 \mathrm{~s}}$ & Cobb $_{2 L 1 s-L 5 i}$ & Cobb $_{111 \mathrm{~s}-\mathrm{S1s}}$ & $\mathrm{Cobb}_{4112 i-\mathrm{S} 1 \mathrm{~s}}$ & Centroid & Posterior ${ }_{\mathrm{L} 1-\mathrm{L} 5}$ & Posterior ${ }_{\text {L1-S1 }}$ & Trall \\
\hline Mean & 50.38 & 41.98 & 55.41 & 58.14 & 35.54 & 29.33 & 60.52 & 35.14 \\
\hline Standard deviation & 9.83 & 10.80 & 10.68 & 9.59 & 11.20 & 9.54 & 11.64 & 7.54 \\
\hline Far below & $<30.72$ & $<20.38$ & $<34.05$ & $<38.96$ & $<13.13$ & $<10.24$ & $<37.24$ & $<20.06$ \\
\hline Moderately below & 30.72 to 40.55 & 20.38 to 31.18 & 34.05 to 44.73 & 38.96 to 48.55 & 13.13 to 24.34 & 10.24 to 19.78 & 37.24 to 48.88 & 20.06 to 27.60 \\
\hline Average & 40.56 to 60.20 & 31.19 to 52.78 & 44.74 to 66.08 & 48.56 to 67.74 & 24.35 to 46.74 & 19.79 to 38.87 & 48.89 to 72.16 & 27.61 to 42.68 \\
\hline Moderately above & 60.21 to 70.03 & 52.79 to 63.58 & 66.09 to 76.76 & 67.75 to 77.33 & 46.75 to 57.95 & 38.88 to 48.42 & 72.17 to 83.80 & 42.69 to 50.22 \\
\hline Far above & $>70.03$ & $>63.58$ & $>76.76$ & $>77.33$ & $>57.95$ & $>48.42$ & $>83.80$ & $>50.22$ \\
\hline
\end{tabular}

Table 4. Kappa statistics between lumbar curvature methods

\begin{tabular}{|c|c|c|c|c|c|c|c|c|}
\hline & $\mathrm{Cobb}_{1 \mathrm{~L} 1-\mathrm{S} 1}$ & $\mathrm{Cobb}_{2 \mathrm{~L} 1-\mathrm{L} 5}$ & $\mathrm{Cobb}_{3 \mathrm{~L} 2 \cdot \mathrm{S} 1}$ & $\mathrm{Cobb}_{4 \mathrm{~T} 12 \cdot \mathrm{S} 1}$ & Centroid & Posterior L1-L5 & Posterior L1-S1 & Trall \\
\hline \multirow{2}{*}{$\mathrm{Cobb}_{1}$} & & 0.634 & 0.351 & 0.491 & 0.322 & 0.052 & 0.366 & 0.215 \\
\hline & & Strong & Acceptable & Moderate & Acceptable & Slight & Acceptable & Acceptable \\
\hline \multirow{2}{*}{$\mathrm{Cobb}_{2}$} & & & 0.401 & 0.545 & 0.503 & 0.113 & 0.344 & 0.315 \\
\hline & & & Acceptable & Moderate & Acceptable & Slight & Acceptable & Acceptable \\
\hline \multirow{2}{*}{$\mathrm{Cobb}_{3}$} & & & & 0.345 & 0.231 & 0.198 & 0.427 & 0.247 \\
\hline & & & & Acceptable & Acceptable & Slight & Moderate & Acceptable \\
\hline \multirow{2}{*}{$\mathrm{Cobb}_{4}$} & & & & & 0.260 & 0.159 & 0.395 & 0.240 \\
\hline & & & & & Acceptable & Slight & Acceptable & Acceptable \\
\hline \multirow{2}{*}{ Centroid } & & & & & & 0.001 & 0.140 & 0.241 \\
\hline & & & & & & Slight & Slight & Acceptable \\
\hline \multirow{2}{*}{ Posterior $_{\text {L1-L5 }}$} & & & & & & & 0.241 & 0.452 \\
\hline & & & & & & & Acceptable & Moderate \\
\hline \multirow{2}{*}{ Posterior ${ }_{\mathrm{L} 1-\mathrm{S} 1}$} & & & & & & & & 0.033 \\
\hline & & & & & & & & Slight \\
\hline
\end{tabular}

vertebral body were compared and, from the moment of results inversion, i.e. after the angular values are no longer increasing or decreasing and reverse at a given vertebra, the inflection point (IP) was located, as evidenced in Figure 1.

It can be observed in the radiographic image (Figure 1) that the identification of the inflection point is very discreet, making it difficult to perform the procedure at the clinic. For the vertebrae T9, T10 and T11, low frequency percentage of the inflection point were found. (Table 5)

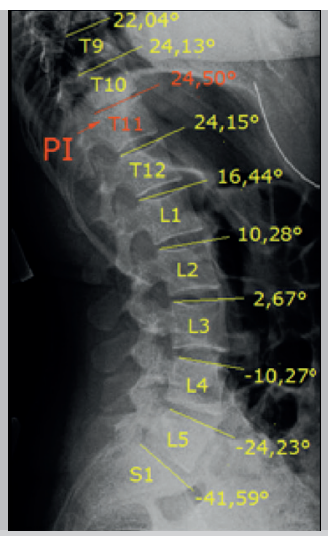

Figure 1. Procedure for detecting the inflexion point of the thoracolumbar spine.

\section{DISCUSSION}

The results show high correlation coefficients among variations in Cobb method: $\mathrm{Cobb}_{4 \text { T12i-S1s }}$ e Cobb 1L1s-S1s $_{\text {( }}(0,893), \mathrm{Cobb}_{4 \text { T12i-S1s }} \mathrm{e}$ $\mathrm{Cobb}_{2 \text { L1s-LSi }}(0,820), \mathrm{Cobb}_{3 \text { L2s-S1s }}$ and $\mathrm{Cobb}_{1 \text { L1s-S1s }}(0,810)$. In these methods, firstly, two dividing lines parallel to the surface of the verte-
Table 5. Descriptive statistics of the inflexion points on the thoracolumbar curvature.

\begin{tabular}{c|c|c}
\hline Vertebra & \% & $\mathbf{N}$ \\
\hline T9 & 1.9 & 1 \\
\hline T10 & 3.9 & 2 \\
\hline T11 & 9.6 & 5 \\
\hline T12 & 38.5 & 20 \\
\hline L1 & 25.0 & 13 \\
\hline L2 & 21.1 & 11 \\
\hline Total & 100.0 & 52 \\
\hline
\end{tabular}

bral bodies are marked, selected in each method and subsequently crossed by two perpendicullar lines, which result in the angles, as shown. 5,6,9-12 (Figure 2)

Despite being widely used in clinics for diagnosis of spine pathologies, we did not identify in the literature review studies that compared all four methods. However, some studies prioritized comparing the reliability of the methods Cobb ${ }_{\mathrm{L1}-\mathrm{S} 1}$ and Cobb ${ }_{L 1-L 5}$, the results indicated interclass correlation coefficients (ICC) of 0.96 and 0.97, for Cobb L1-S1 and Cobb L1-L5 intraobserver and values of 0.95 and 0.96 for Cobb L1-S1 and Cobb $_{\text {L1-L5 }}$ interobservers, respectively. These facts corroborate the strong correlative evidence also found in this study. ${ }^{6}$ The purpose of comparing those Cobb methods emerges from the failure in obtaining radiographic image, because defects such as blurring, granulation, dimming or overlaping organs in the areas studied may compromise the identification of anatomical reference points of the selected test, thus hindering to obtain the correct measurement point.

The centroid method, also used in this study, allows making marks that follow the actual contour of the vertebral bodies, 
therefore, decreasing the negative influence caused by the irregularity of the vertebrae in angular measurements. ${ }^{5}$ (Figure 3) From this theory, Chen ${ }^{13}$ compared the centroid method, with two tests: $\mathrm{Cobb}_{\mathrm{L1}-\mathrm{L} 5}$ and $\mathrm{Cobb}_{\mathrm{L} 1-\mathrm{S} 1}$. The results of inter and intraobservation with three different evaluators showed that, among all, the centroid method had the highest average interclass correlation,

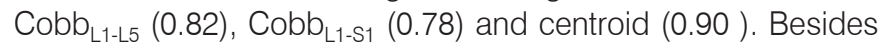
the correlative approach of the centroid method compared to other tests present in this sample, it was possible to identify through categorization, the reference angle values for a population sample of the Southern region of Brazil. This contribution defines a framework of angle values, considering the absence of such data in the current literature.

The posterior tangent method (Figure 4) uses a traced line that demarcates the extension of the posterior wall of the vertebral body of $\mathrm{L} 1$ and another indicating S1. The intersection of the two lines derived from later portions of the aforemenined vertebrae generate the angular measure. Lee et al. ${ }^{6}$ found that the measurement of the posterior tangent angle is more reproducible, because it showed the highest rate of reliability in comparisons to $\mathrm{Cobb}_{\mathrm{L} 1-\mathrm{S} 1}$. In this study, there was a moderate correlation $(r=0.63)$ between these two methods. The moderate result can be explained by the fact that both methods use the same anatomical points, L1-S1. Despite the geometric patterns are different, it is considered as a priority intervening the positioning of the vertebral body, as described in Chen's work. ${ }^{13}$ The Trall method was designed from the intersection of straight

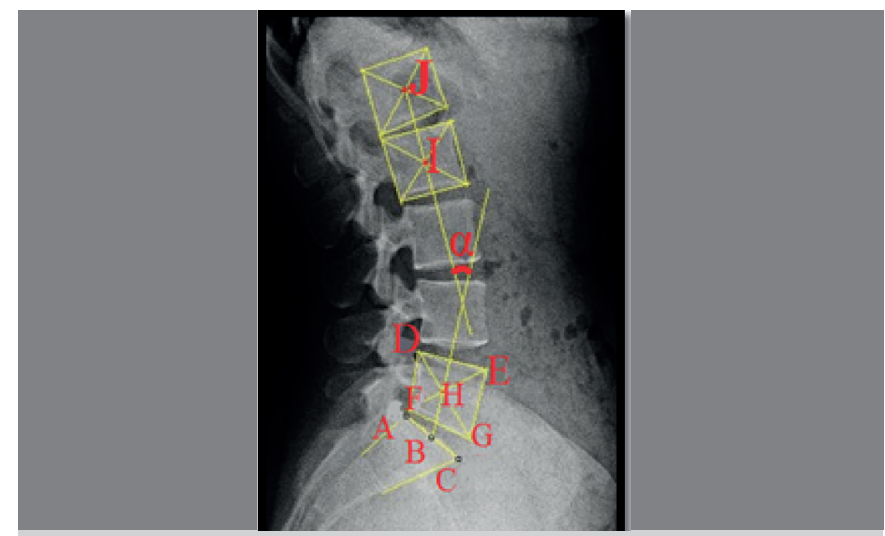

Figure 2. Procedure for performing the centroid method.

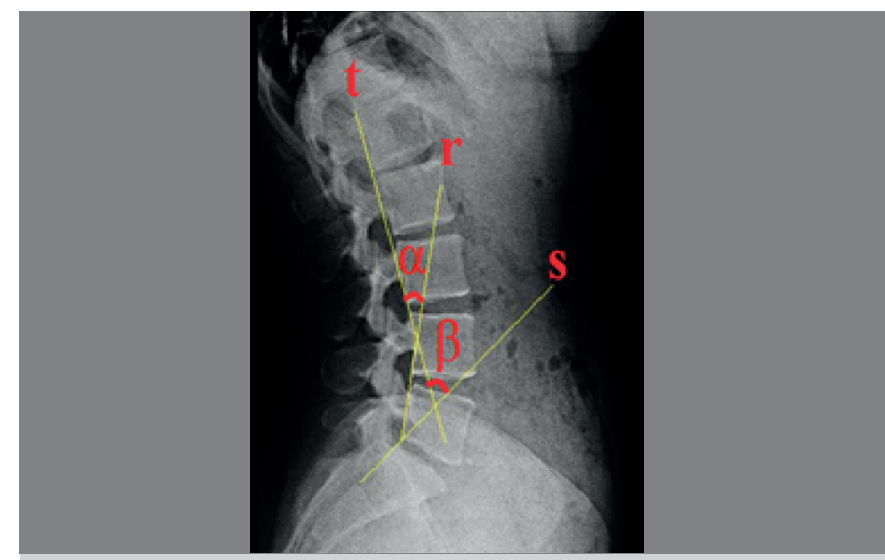

Figure 3. Procedure for performing the posterior tangent method. lines that run from posterosuperior vertex L1 vertebra and the posterior-vertex of S1 vertebra, crossing over the point of greatest depth of lordosis, ie, the point of greatest distance from the line passing on the vertices. (Figure 5) The origin of this measure came in order to override the method of variations, so existing, Cobb. In addition, it presents greater reproducibility, with the design of differential curvature of the lumbar spine and suffers less variations of the vertebral bodies in its contradiction medida.6,14,15 in this study there was a strong correlation $(r=0.70)$ between the method of Trall and the methodology of Cobb2 L1s-L5i. This result can be explained by the influence of the $\mathrm{L} 1$ vertebra Furthermore, comparisons with other methods showed moderate Cobb correlations.

As for categorical classification it was not possible to identify the reference values indicated for each method studied, except for the Cobb $_{\text {L1-L5 }}$ method, whose reference values range from 40 to 60 degrees. ${ }^{16}$ From this fact, it was noted the necessity for a categorization for fundamented assessment to the other methods. The relations obtained from the Kappa agreement results showed correlative indexes of 0.63 for the angles $\mathrm{Cobb}_{1}$ and $\mathrm{Cobb}_{2}$ between moderate measures. $\mathrm{Cobb}_{4}$ method when compared to $\mathrm{Cobb}_{1}$ and $\mathrm{Cobb}_{2}$ reached indices of 0.49 and 0.54 , respectively. These findings show that the measurements performed with the same angular pattern show proven stronger relations. ${ }^{13}$ Possibly those results rated as reasonable and weak indicate the discrepancy between the measurement points, and analyzing this reason the identification of the inflection point is discussed.

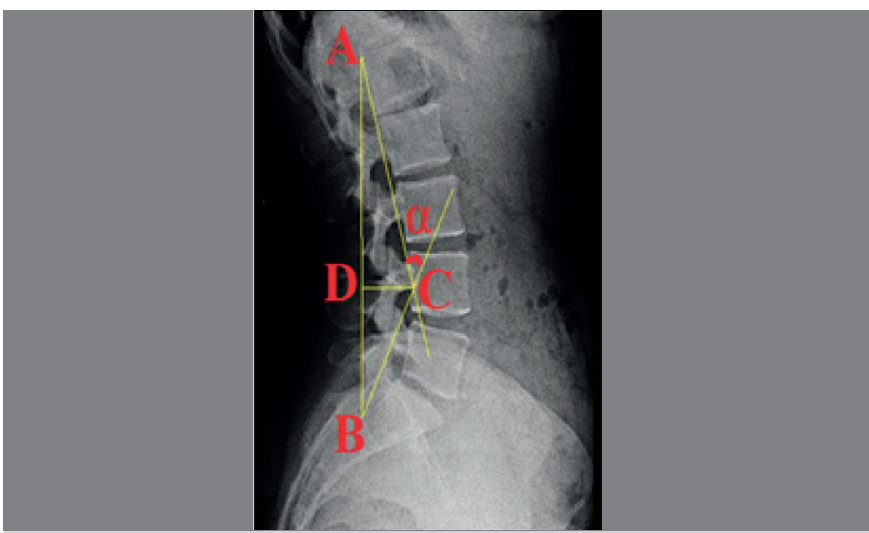

Figure 4. Procedure for performing the Trall method.

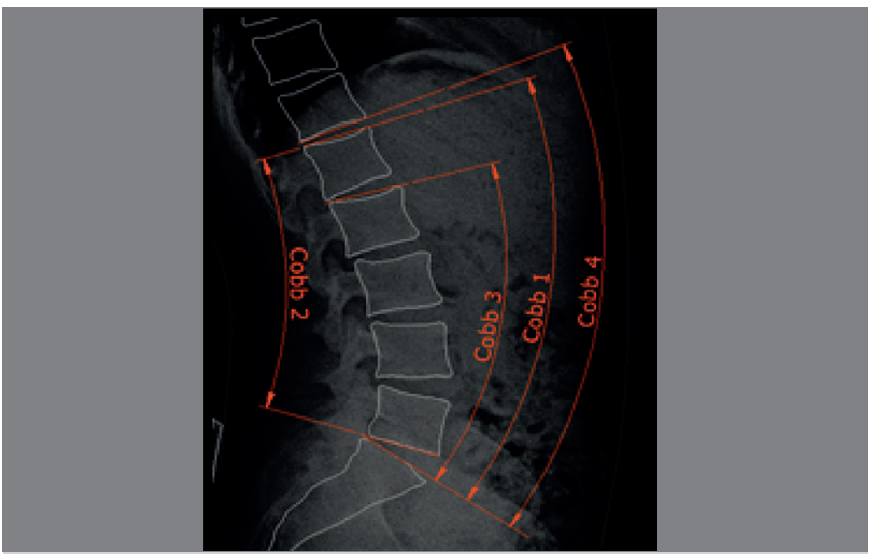

Figure 5. Trace of angles $\mathrm{Cobb}_{1 \mathrm{L1}-\mathrm{S} 1}, \mathrm{Cobb}_{2 \mathrm{L1}-\mathrm{L} 5}, \mathrm{Cobb}_{3 \mathrm{L2}-\mathrm{S} 1}$ and $\mathrm{Cobb}_{4 \mathrm{T12}-\mathrm{S} 1}$. 
Then, it has been shown that in the current sample, the vertebra occurring in the inflection point which appeared more frequently was $T 12$, with $38.5 \%(n=20)$. In the case of vertebrae $L 1$ and $\mathrm{L} 2$, the occurrence of the inflection point occurred at $25.0 \%$ $(n=13)$ and $21.1 \%(n=11)$ of patients, respectively.

The identification of the inflection point of the thoracolumbar spine, and its influences are not widely used in the present study, which focused the structure of the backbone, although there are investigative work, as proposed by Singer et al., ${ }^{17}$ who reported the presence of the inflection point between vertebrae T10 and $\mathrm{T} 12$ in 286 sagittal $\mathrm{X}$-rays. Other evidences regarding studies that have suggested that, besides determining the inflection point in the coordinate system of the spine showed that, in addition to determining more accurately the start of the lumbar curvature, this point may help the quantitative analysis of the lumbar curvature in two planes simultaneously (coronal and sagittal). ${ }^{18}$

For example, to the vertebrae above T12, the angular Trall test should be applied. As for the inflection points found between T12 and L1, the methods Cobb ${ }_{1 \text { L1s-S1S }}$; $\mathrm{Cobb}_{2 \text { L1s-L5i; }}$ and posterior tangent $\mathrm{L} 1 \mathrm{~L}-\mathrm{L}_{\mathrm{C}}$ can be recommended for demarcation. For the identification of the inflection point from the second lumbar vertebra the, $\mathrm{Cobb}_{3 \text { L2s-L1s }}$ methods are indicated. Finally, in all participants presenting the inflection point below the L2 vertebra the centroid method is recommended for viabilizing measurement at any point of inflection.

To verify the effectiveness of the influence of the inflection point, there was a correlation between the techniques indicated in the same inflection location. As for vertebrae T12 and L1 more than one technique was indicated, which showed a moderate to strong correlation between them.

\section{REFERENCES}

1. Le Huec JC, Charosky S, Barrey C, Rigal J, Aunoble S. Sagittal imbalance cascade for simple degenerative spine and consequences: algorithm of decision for appropriate treatment. Eur Spine J. 2011;20(Suppl 5):699-703.

2. Thomas KS, VanLunen BL, Morrison S. Changes in postural sway as a function of prolonged walking. Eur J Appl Physiol. 2013;113(2):497-508.

3. Dawson AP, Schluter PJ, Hodges PW, Stewart S, Turner C. Fear of movement, passive coping, manual handling, and severe or radiating pain increase the likelihood of sick leave due to low back pain. Pain. 2011;152(7):1517-24.

4. Morvan G, Mathieu P, Vuillemin V, Guerini H, Bossard P, Zeitoun F. Standardized way for imaging of the sagittal spinal balance. Eur Spine J. 2011;20(Suppl 5):602-8.

5. Hwang JH, Modi HN, Suh SW, Hong JY, Park YH, Park JH, et al. Reliability of lumbar lordosis measurement in patients with spondylolisthesis: a case-control study comparing the Cobb, centroid, and posterior tangent methods. Spine (Phila Pa 1976). 2010;35(18):1691-700.

6. Lee JS, Goh TS, Park SH, Lee HS, Suh KT. Radiographic measurement reliability of lumbar lordosis in ankylosing spondylitis. Eur Spine J. 2013;22(4):813-8.

7. Hinton RP, Brownlow C, McMurray I, Cozens B. SPSS Explained. New York: Routledge; 2004.

8. Landis JR, Koch GG. The measurement of observer agreement for categorical data. Biometrics. 1977;33(1):159-74.

9. Kwok AW, Wang YX, Griffith JF, Deng M, Leung JC, Ahuja AT, et al. Morphological changes of lumbar vertebral bodies and intervertebral discs associated with decrease in bone mineral density of the spine: a cross-sectional study in elderly subjects. Spine (Phila Pa 1976). 2012;37(23):E1415-21.
Thus, Table 6 shows a suggestion of which methods are the most appropriate for each individual, in accordance with the inflection point region.

Table 6. Classification defined to division of anatomical regions and respective recommended methods.

\begin{tabular}{|c|c|c|}
\hline Vertebra classification & Indicated methods & Percentage of the sample \\
\hline Above T12 & Trall & $15.4 \%$ \\
\hline \multirow{4}{*}{$\mathrm{T} 12-\mathrm{L} 1$} & $\mathrm{Cobb}_{4}$ T12i-S1s & \multirow{4}{*}{$63.5 \%$} \\
\hline & Cobb $_{1 \text { L1s-S1s }}$ & \\
\hline & $\mathrm{Cobb}_{2 \text { L1s-L5i }}$ & \\
\hline & Posterior $_{\text {L1-L5 }}$ & \\
\hline L2 & $\mathrm{Cobb}_{3 \text { L2s-S1s }}$ & $21.1 \%$ \\
\hline Below L2 & Centroid & $0.0 \%$ \\
\hline Total & & $100.0 \%$ \\
\hline
\end{tabular}

\section{CONCLUSION}

It was possible to identified with this study that the correlative and agreement values were most significant for the Cobb LL1-S1 $_{1}$ $\mathrm{Cobb}_{2 \mathrm{L1}-\mathrm{L} 5}, \mathrm{Cobb}_{3 \mathrm{L2}-\mathrm{S} 1}$ and $\mathrm{Cobb}_{4 \mathrm{~T} 12-\mathrm{S} 1}$ methodology. However, we also observed a strong correlation $(r=0.70)$ between $\mathrm{Cobb}_{2}$ and Trall methods. Therefore, it can be concluded that the diversity of anatomical references used in the studied techniques should be considered when choosing a techique to a given patient, depending on the placement of the thoracolumbar inflection point, since knowledge of its location allows the choice of technique to be used more accurately.

10. Fernand R, Fox DE. Evaluation of lumbar lordosis. A prospective and retrospective study. Spine (Phila Pa 1976). 1985;10(9):799-803.

11. Lord MJ, Small JM, Dinsay JM, Watkins RG. Lumbar lordosis. Effects of sitting and standing. Spine (Phila Pa 1976). 1997;22(21):2571-4.

12. Chang MS, Bridwell KH, Lenke LG, Cho W, Baldus C, Auerbach JD, et al. Predicting the outcome of selective thoracic fusion in false double major lumbar "C" cases with five- to twenty-four-year follow-up. Spine (Phila Pa 1976). 2010;35(24):2128-33.

13. Chen YL. Vertebral centroid measurement of lumbar lordosis compared with the Cobb technique. Spine (Phila Pa 1976). 1999;24(17):1786-90.

14. Harrison DE, Harrison DD, Cailliet R, Janik TJ, Holland B. Radiographic analysis of lumbar lordosis: centroid, Cobb, TRALL, and Harrison posterior tangent methods. Spine (Phila Pa 1976). 2001; 26(11):E235-42.

15. Hong JY, Suh SW, Modi HN, Hur CY, Song HR, Park JH. Reliability analysis for radiographic measures of lumbar lordosis in adult scoliosis: a case-control study comparing 6 methods. Eur Spine J. 2010;19(9):1551-7.

16. Kapandji IA. Fisioterapia articular: tronco e coluna vertebral. 5a. ed. Rio de Janeiro: Guanabara; 2000

17. Singer KP, Jones TJ, Breidahl PD. A comparison of radiographic and computer-assisted measurements of thoracic and thoracolumbar sagittal curvature. Skeletal Radiol. 1990;19(1):21-6.

18. Campos $\mathbf{M H}$. Sistema de análise de movimento para avaliação da postura vertebral durante a corrida no teste de esforço máximo incremental [tese]. Campinas: Universidade Estadual de Campinas; 2010. 